\title{
Roles of adiponectin and leptin as diagnostic markers in pancreatic cancer
}

\author{
Kadri Colakoglu M, Bostanci EB, Ozdemir Y, Dalgic T, Aksoy E, Ozer I, Ozogul Y, Oter V \\ Department of Gastroenterology Surgery, Ankara Yukses Ihtisas Training and Research Hospital, \\ Turkiye Yuksek Ihtisas Teaching and Research Hospital, Ankara, Turkey. otervolkan@gmail.com
}

\begin{abstract}
INTRODUCTION: Obesity is one of the most serious public health problem worldwide. Adipose tissue synthetize and secrete many growth factors and several cytokines known as adipokines. Studies demonstrated changes in the levels of these adipokines in many types of cancer associated with obesity.

In this study, we aimed to evaluate the possible relationship between adiponectin and leptin levels with pancreas cancer and disease stage, representative of Turkish population.

MATERIALS AND METHODS: The study was conducted between April 2012 - November 2013. Study included 46 patients - 46 control subjects, who had pancreatic carcinoma. Results between the patients and the control group and relationship between the disease stage and results were evaluated.

RESULTS: The comparison of preoperative adiponectin and leptin levels of the study group with the levels of the control group showed that there was no correlation with adiponectin and pancreas cancer. In contrast, leptin levels in the study group were significantly lower than in the control group. There was no correlation between the disease stage and adiponectin and leptin levels.

CONCLUSION: There was a significant correlation between low leptin levels and pancreatic cancer, while adiponectin had no correlation. Differential diagnosis of pancreas cancer can be made by evaluating low leptin levels with elevated tumor markers (Tab. 3, Ref. 17). Text in PDF www.elis.sk.
\end{abstract}

\section{Introduction}

Obesity is one of the most serious public health problem in both developed and developing countries throughout the world. The prevalence has increased dramatically in recent years and has even become endemic in some communities. Similarly, cancer is the leading cause of death in developed countries, while in developing countries it is the second most frequent. Studies that showed the relationship between these two leading health problems are increasing in number and their results revealed strong epidemiologic and molecular evidences that obesity is a risk factor in development of many cancer types.

Most of the research about the relationship between obesity and cancer is based on the fact, that adipose tissue is an endocrine organ. During obesity, adipose tissue mass in the body increases. Adipose tissue synthetize and secrete many growth factors and several cytokines known as adipokines. Adipokines are mainly produced by white adipose tissue, pre-adipocytes and mature adipocytes. It has been shown that many adipokines modify the metabolic cellular functions in rodents and humans.

Leptin and adiponectin, which are the significant members of the adipokine family, might play important roles in the patho-

Address for correspondence: V. Oter, Department of Gastroenterological Surgery, Turkiye Yuksek Ihtisas Teaching and Research Hospital, Atatürk Boulevard, Kizilay Street, No: 4 Sihhiye, Ankara, 06230 Turkey.

Phone: +905053366333, Fax: +903123100378 genesis of obesity-related cancers. The studies referred that changing leptin and adiponectin levels is associated with various types of cancer of the gastrointestinal tract. Experimental studies demonstrated that both have significant effects on cell proliferation, apoptosis, cell invasion and angiogenesis. Based on these effects, studies showed that in many types of cancer, particularly colorectal, breast and prostate, the levels of these cytokines differ from normal individuals and even molecular mechanism of this effect has been understood in many cancer types.

Pancreatic cancer is one of the deadliest type of cancer in adults. Many patients are in advanced stage at the time of diagnosis. Greatest dilemma of the disease is the non-specific symptoms that can be kept confidential for many years. A diagnostic test with high sensitivity and specificity for early diagnosis has not yet been established. Increased body mass has been shown as a risk factor for pancreatic cancer in epidemiological studies. In the light of this information, the relationship between, adipokines secreted by adipose tissue and pancreatic cancer started to be questioned. In many studies performed, adiponectin blood levels indicated a negative correlation, while leptin levels showed a positive correlation with obesity-related cancers. These correlations were confirmed in many studies considering body mass index (BMI) and insulin resistance.

In this study, we aimed to determine the blood levels of adiponectin and leptin in patients with pancreatic cancer and evaluated the possible relationship between the results and disease stage representative of Turkish population. 


\section{Materials and methods}

After literature review and preliminary investigations were made, the research project was prepared and submitted to the ethical committee of Ankara Yuksek Ihtisas Hospital, Turkey, where the study was performed. The study started in April 2012 after the approval was obtained.

\section{Study group}

Between April 2012 and November 2013, files of pancreatic cancer patients, who were newly diagnosed and prepared for surgery in the Department of Gastroenterology Surgery, Ankara Yuksek Ihtisas Hospital were reviewed. Patients with history of different malignancies, or pancreatic endocrine tumors and patients, who received neoadjuvant therapy were excluded from the study. Totally, 72 patients, who met these criteria, were evaluated. Data about age, gender, BMI, preoperative carbohydrate antigen 19-9 (CA 19-9) and carcinoembryonic antigen (CEA) levels, smoking and alcohol history, weight loss, history of diabetes, family history of malignancy and operation performed were recorded prospectively by questioning the patients and reviewing of the files.

Ten cubic centimeters (cc) of venous blood was obtained from the patients the night before or the morning on the day of operation and kept in biochemistry tube. After keeping these tubes at room temperature for about 15-30 minutes, they were centrifuged for 15 minutes at 2,500 rpm in centrifuge. Blood serum obtained was placed to empty plastic storage tubes, and the remaining cell suspension was discarded. Printed labels with patient's name, surname and protocol numbers written on it were pasted on the surface of these transparent storage tubes. The tubes were collected in a storage container and kept in a special refrigerator that provided coldness at -75 centigrade degree $\left({ }^{\circ} \mathrm{C}\right)$.

After the patient was operated, on the 7 th postoperative day, the serum was obtained for the second time using the method described and these tubes were kept at the same storage conditions and stored in different containers for evaluating the postoperative values. If the patients were evaluated as unresectable or metastatic during operation, postoperative bloods were not obtained. Also, postoperative evaluation could not be done if the patient died within 7 postoperative days.

Postoperative pathology results of these 72 patients followed and 46 patients, whose primary pathology were pancreatic malignancy were determined and the stage of pancreatic cancer of these patients were recorded. These 46 patients formed the study group. The remaining 26 patients with postoperative pathology results such as: periampullary tumor, benign lesions or neuroendocrine tumors were excluded from the study.

\section{Control group}

Between April 2012 and November 2013, files of cholelithiazis patients, who were newly diagnosed and prepared for surgery in the same hospital, were reviewed. Patients with a history of previous malignancy, acute cholecystitis, choledocholithiasis or pancreatitis were excluded from the study. Data from the same number of patients (46 patients) as in the study group were evaluated. Data about age, gender, BMI, smoking and alcohol history, history of diabetes and family history of malignancy were recorded prospectively. Blood serum was obtained from the control patients with the method described earlier and samples were kept at the same storage conditions and stored in different containers.

\section{Obtaining data}

At the end of the study, preoperative and postoperative serum samples of the study group and serum samples of the control group were kept at $-4{ }^{\circ} \mathrm{C}$ the night before the biocheminal evaluation, for minimal dissolving of the samples. The samples were then moved to the biochemical laboratory following the rules of cold chain. After the samples dissolved in room temperature, biochemical studies were performed on the same day. Biovender ELISA kit (Czech Republic) used for biochemical study of adiponectin and results were given as $\mu \mathrm{g} / \mathrm{mL}$ and Diasource ELISA kit (Belgium) used for biochemical study of leptin and results were given as ng/ $\mathrm{mL}$. Both ELISA kits were evaluated by Biotek ELISA Synergy HT reader model.

Statistics

Descriptive characteristics of the study and control groups were expressed as the mean \pm standard deviation (SD), unless indicated otherwise. Minimum and maximum values were provided when necessary. Komolgornov-Smirnov test was used to assess the distribution of the data. The homogeneity of variance was evaluated by Levene test. Analysis of continuous variables between cases and the control group were performed by paired t-test. In the analysis of classified data, chi-square test was used. The significance of the difference between median values of clinic measurements of day 1 and the study group, and materiality of the difference in median values in clinical measurements made on day 7 was investigated by One-Way ANOVA test. The relationship between stage, tumor markers and adipokines was done with Two-Tailed Pearson correlation test. The $\mathrm{p} 0.05$ value was considered to be significant. Statistical analysis was accomplished using the SPSS program for Windows 22.0 (SPSS, Chicago, IL, United States).

\section{Results}

This study was conducted between April 2012 and November 2013. Demographic data and preoperative and postoperative adiponectin and leptin levels of 46 patients with primary pancreatic

Tab. 1. The demographic characteristics of patients in study and control groups.

\begin{tabular}{lccc}
\hline $\begin{array}{l}\text { Demographic } \\
\text { characteristics }\end{array}$ & Study group & Control group & $\mathrm{p}$ \\
\hline Gender & Male: 29 & Male: 16 & 0.004 \\
\hline Age (year) & Female: 17 & Female: 30 & \\
BMI $\left(\mathrm{kg} / \mathrm{m}^{2}\right)$ & $60.98 \pm 13.26$ & $55.42 \pm 14.22$ & 0.128 \\
Smoking $(\%)$ & $22.63 \pm 2.75$ & $24 \pm 3.5$ & 0.917 \\
Alcohol $(\%)$ & 45.7 & 46 & 0.973 \\
Diabetes $(\%)$ & 17.4 & 46 & 0.003 \\
\hline
\end{tabular}


Tab. 2. Evaluation of adipokines between study group and control group.

\begin{tabular}{lccc}
\hline Adipokines & Study group & Control group & $\mathrm{P}$ \\
\hline Adiponectin & $9.97 \pm 4.67$ & $9.67 \pm 4.93$ & 0.677 \\
Leptin & $6.92 \pm 8.66$ & $7.36 \pm 7.95$ & 0.035 \\
\hline
\end{tabular}

Tab. 3. Comparison of preoperative and postoperative adiponectin and leptin levels in the study group.

\begin{tabular}{lccc}
\hline Adipokines & Preoperative & Postoperative & $\mathrm{p}$ \\
\hline Adiponectin & $9.97 \pm 4.67$ & $10.52 \pm 6.04$ & 0.04 \\
Leptin & $6.92 \pm 8.66$ & $3.14 \pm 3.81$ & 0.002 \\
\hline
\end{tabular}

malignancy, who were admitted to the Gastroenterology Surgery Department of Yuksek Ihtisas Hospital between these dates were recorded. Between the same dates, demographic data and adiponectin and leptin levels of 46 control patients, who were operated because of cholelithiasis were also recorded.

There was no significant difference according to age, BMI, smoking and diabetes mellitus history between the two groups. However, the two groups showed differences in gender and alcohol use. The number of male patients in the study group was higher, while the number of female patients was higher in the control group. Also, the rate of patients, who had an alcohol history in the control group was higher almost 3 times from the study group. In both groups, there was no family history of cancer in any of the family members. The demographic characteristics of patients and p-values are presented in the Table 1.

When preoperative adiponectin and leptin levels in the study group were compared to the control group, no difference was observed between the two groups in terms of adiponectin. In other words, there was no difference according to the adiponectin levels between the patients, who had a pancreatic cancer diagnosis and who did not. On the contrary, when we look at the level of leptin, leptin levels in the study group were found to be significantly lower than in the control group. In other words leptin levels were significantly lower in the patients, who had a pancretic cancer diagnosis and this was independent of BMI and diabetes mellitus history. Adiponectin and leptin levels with p-values of the study and control groups are presented in the Table 2 .

When staging of patients with pancreatic cancer made, 5 of them were stage 1a, 8 were stage $1 \mathrm{~b}, 6$ were stage $2 \mathrm{a}, 14$ were stage $2 b, 2$ were stage 3 and 11 were stage 4 . Only preoperative adiponectin and leptin values were evaluated in stage 4 and stage 3 patients, who were unresectable at the time of surgery. Three patients from the other stages died before postoperative seventh day. When the preoperative and postoperative adiponectine and leptin levels were evaluated in remaining 30 patients, we found that, adiponectin levels were significantly increased and leptin levels were significantly decreased postoperatively. These values are shown in the Table 3.

We questioned whether the adiponectin and leptin levels showed any differences according to the disease stage. Analysis revealed that adiponectin levels did not indicate any changes according to different disease stages $(p=0.6)$. Also, analysis re- vealed that leptin levels did not change in different disease stages. Leptin levels did not differ statistically according to the stages $(\mathrm{p}=0.42)$.

Tumor marker of patients (CA 19-9/CEA) were also evaluated. Both tumor markers were correlated with the stage of the patients ( $p=0.02$ and $p=0.018$ respectively). When this tumor markers were compared to adiponectin and leptin levels, no correlation was found $(\mathrm{p}>0.05)$.

\section{Discussion}

Adipose tissue is an active endocrine organ that secretes several bioactive adipokines that regulate various physiological and pathological processes $(1,2)$. In literature, the most frequently studied adipokines are leptin and adiponectin. Leptin regulates many physiological functions in humans and mainly adjusts the energy balance. Low leptin levels are significantly associated with insulin resistance. Adiponectin is an insulin-sensitizing, anti-inflammatory and anti-atherogenic adipokine. In vivo studies showed that it has a protecting effect on several types of cancer (3-9).

The literature showed that many cancer types have negative correlation with adiponectin, but positive correlation with leptin levels. The relationship between these two adipokines with pancreatic cancer, which is associated with obesity, is controversial. Study of Chang MC et al in 2007 about usage of serum adiponectin levels in differentiating pancreatic cancer and pancreatitis revealed that adiponectin levels were much higher in patients with pancreatic cancer than in patients with chronic pancreatitis and suggested that high adiponectin levels could be used in the differential diagnosis (10).

This was the first study that tried to show the relationship between pancreatic cancer and adiponectin, but authors suggested that the different results from other cancer types could be due to different molecular mechanisms of adiponectin in pancreatic cancer, cholestasis might increase adiponectin levels and the status of weakness secondary to pancreatic cancer might increase adiponectin levels. In 2008, Stolzenberg-Solomon et al conducted a study, in which patients in the study cohort were followed up and adiponectin levels of patients, who developed pancreatic cancer during follow up time, were evaluated. Consequently, they suggested that high adiponectin concentration was inversely associated with development of pancreatic cancer (protective effect) (11). The strength of this study comes from a high number of cases included and its prospective design, but its unsatisfactory part is that study included only male smokers.

The relationship between adiponectin and leptin together with pancreatic cancer was first evaluated in the case control study, which included Greek population, in 2009 and in addition, a presence of adiponectin receptors in pathology specimens was demonstrated. As a result, unlike other cancer types, it was shown that high adiponectin levels and low leptin levels were associated with pancreatic cancer. In this paper, authors argued that, in early phases of disease it could be predicted that low adiponectin and high leptin levels were associated with obesity, but as a response to insulin resistance and rapid weight loss, as stage of disease in- 
crease, these values can be reversed quickly. Indeed, half of the patients in this study were stage IV (12).

There are studies suggesting that there might be different adipokine responses in different types of cancer. Based on the conflicting results in literature about relationship between adiponectin and pancreas cancer, Phelip JM et al evaluated adiponectin levels of 33 colorectal and 53 pancreatic cancer patients and revealed that adiponectin levels in pancreatic cancer patients were higher than (12 fold) in colorectal patients. Authors revealed that as adipokin levels could be affected by various factors such as BMI, gender and race, it can also be affected by type of the cancer (13). However, this article did not compare the results with the normal population.

The most comprehensive study about adiponectin is the casecontrol study conducted by Grote VA et al. Including 10 European countries with 23 different centers, adiponectin levels of 452 patients with pancreas cancer and same number of control patients from prospective EPIC cohort were evaluated. As a result, they suggested that low adiponectin levels could be associated with the development of pancreatic cancer in non-smokers (14). Similarly, in the study using 5 cohorts and published recently, 468 pancreatic cancer patients were compared to 1080 control patients and suggested that low adiponectin levels were associated with pancreatic cancer independent from gender, smoking and BMI (15). As seen, first studies about this subject showed a direct relationship between high adiponectin and low leptin levels with pancreatic cancer, but with the inclusion of large cohort studies and increase in the number of cases, this relationship turned inversely, especially for adiponectin.

This study found that leptin levels were lower in pancreatic cancer patients according to control population and this decrease was statistically significant. This result is, in fact, consistent with other literature results related to pancreatic cancer and leptin. Although it is a cancer type associated with obesity, it remains controversial, why leptin levels decreased in pancreatic cancer. Hypoleptinemia may have arisen as the result of weight loss seen in many cancer patients. In this study, the mean BMI was $22 \mathrm{~kg} / \mathrm{m}^{2}$, but it is to be noted that $60 \%$ of patients had a history of weight loss.

Another argument is the different molecular effect of leptin on pancreas. In vitro studies of leptin showed that it inhibited the growth of two human pancreatic cancer cell lines (PANC-1, $\mathrm{Mia}-\mathrm{PaCa}$ ) and it was probably managed by its proimmunogenic effects (16). Proliferation of cancer cell lines might be provided with a lack of leptin.

Pezzill $\mathrm{R}$ et al evaluated leptin and adiponectin as a distinctive marker for chronic pancreatitis, autoimmune pancreatitis and pancreatic cancer (17). In their study with 75 patients, they revealed that leptin was significantly low in chronic pancreatitis and pancreatic cancer, but increased in autoimmune pancreatitis. They suggested that low leptin levels with high CA-19-9 levels could be used as a distinctive marker for pancreatic cancer. Similarly, leptin levels were low and CA-19-9 levels were correlated with disease stage in our study and adiponectin levels did not differ from control population.

When interpreting studies about adiponectin, discordance about epidemiology and design between studies should be kept in mind. Case-control studies demonstrated a relationship between high levels of adiponectin with pancreas cancer, but prospective studies demonstrated opposite. Pezzillis' work and our study found no correlation between them. As mentioned before, low adiponectin level could be significant because of its well known protective effect. On the other hand, increase of adiponectin levels in prolonged weight loss and anorexia nervosa might explain the rise of adiponectin in cachexia due to pancreatic cancer. It should be kept in mind that, adiponectin may also increase as a compensation to insulin resistance, which is very common in pancreatic cancer. Therefore, studies aimed to understand the molecular mechanisms of adiponectin associated with pancreatic cancer or designing metaanalysis about adiponectin level will shed more light on this issue.

As to our knowledge, literature does not include any studies about relationship between adiponectin and leptin levels with pancreatic cancer stage. In our study, we found no correlation between disease stage and plasma levels of adiponectin and leptin. This situation raises doubts about that adiponectin and leptin are not related with disease progression, but involve in the mechanisms of disease onset. Also, it is very interesting that preoperative and postoperative values of both adiponectin and leptin levels in the study group are significantly different. Adiponectin levels increase, while leptin levels decrease postoperatively in pancreatic cancer patients. It is necessary to clarify the mechanism.

Another impasse of this study is the difference of some factors between patients and controls. As an example, alcohol usage was much higher in the control group. Effect of alcohol on pancreas cancer is not clear and there are no studies suggesting that adipokine levels can be changed with the use of alcohol. Nevertheless, it will be helpful to design the study taking this into account. In the same way, distribution of men and women between the patients and the control group were not equal. It is thought that adiponectin and leptin levels might vary with gender. However, to support this thesis, adiponectin and leptin levels should be evaluated in normal individuals of same race to show difference between male and female gender. Nevertheless, it would be helpful to consider these factors to provide homogeneity.

\section{Conclusion}

As the result, there was a significant correlation between low leptin levels and pancreatic cancer, while adiponectin had no correlation. Differential diagnosis of pancreas cancer could be made by evaluating low leptin levels with elevated tumor markers.

\section{References}

1. Matarese G, Mantzoros C, La Cava A. Leptin and adipocytokines: bridging the gap between immunity and atherosclerosis. Curr Pharm Des 2007; 13: 3676-3680.

2. Kershaw EE, Flier JS. Adipose tissue as an endocrine organ. J Clin Endocrinol Metab 2004; 89: 2548-2556.

3. Mantzoros C, Petridou E, Dessypris N et al. Adiponectin and breast cancer risk. J Clin Endocrinol Metab 2004; 89: 1102-1107. 


\section{4-398}

4. Dal Maso L, Augustin LS, Karalis A et al. Circulating adiponectin and endometrial cancer risk. J Clin Endocrinol Metab 2004; 89: 1160-1163.

5. Goktas S, Yilmaz MI, Caglar K, Sonmez A, Kilic S, Bedir S. Prostate cancer and adiponectin. Urology 2005; 65: 1168-1172.

6. Ishikawa M, Kitayama J, Kazama S, Hiramatsu T, Hatano K, Nagawa H. Plasma adiponectin and gastric cancer. Clin Cancer Res 2005; 11 : 466-472.

7. Spyridopoulos TN, Petridou ET, Skalkidou A, Dessypris N, Chrousos GP, Mantzoros CS. Low adiponectin levels are associated with renal cell carcinoma: a case-control study. Int J Cancer 2007; 120: 1573-1578.

8. Wei EK, Giovannucci E, Fuchs CS, Willett WC, Mantzoros CS. Low plasma adiponectin levels and risk of colorectal cancer in men: a prospective study. J Natl Cancer Inst 2005; 97: 1688-1694.

9. Mantzoros CS, Trakatelli M, Gogas $\mathbf{H}$ et al. Circulating adiponectin levels in relation to melanoma: a case-control study. Eur J Cancer 2007; 43: 1430-1436.

10. Chang MC, Chang YT, Su TC et al. Adiponectin as a potential differential marker to distinguish pancreatic cancer and chronic pancreatitis. Pancreas 2007; 35: 16-21.

11. Stolzenberg-Solomon RZ, Weinstein S, Pollak M et al. Prediagnostic adiponectin concentrations and pancreatic cancer risk in male smokers. Am J Epidemiol 2008; 168 (9): 1047-1055
12. Dalamaga M, Migdalis I, Fargnoli JL, Papadavid E, Bloom E, Mitsiades N, Karmaniolas K, Pelecanos N, Tseleni-Balafouta S, Dionyssiou-Asteriou A, Mantzoros CS. Pancreatic cancer expresses adiponectin receptors and is associated with hypoleptinemia andhyperadiponectinemia: a case-control study. Cancer Causes Control 2009; 20 (5): 625-633.

13. Phelip JM, Bageacu S, Baconnier M, Barabino G, Del Tedesco E, Benhamou PY, Roblin X. Comparison of adiponectin concentration between pancreatic cancer and colorectal cancer. J Gastrointest Oncol 2011; 2 (4): 232-239.

14. Grote VA, Rohrmann S, Dossus L et al. The association of circulating adiponectin levels with pancreatic cancer risk: a study within the prospective EPIC cohort. Int J Cancer 2012; 15; 130 (10): 2428-2437.

15. Bao Y, Giovannucci EL, Kraft $P$ et al. A prospective study of plasma adiponectin and pancreatic cancer risk in five US cohorts. J Natl Cancer Inst 2013; 105 (2): 95-103.

16. Somasundar P, Yu AK, Vona-Davis L, McFadden DW. Differential effects of leptin on cancer in vitro. J Surg Res 2003; 113 (1): 50-55.

17. Pezzilli R, Barassi A, Corsi MM, Morselli-Labate AM, Campana D, Casadei R, Santini D, Corinaldesi R, D’Eril GM. Serum leptin, but not adiponectin and receptor for advanced glycation end products, is able to distinguish autoimmune pancreatitis from both chronic pancreatitis and pancreatic neoplasms. Scand J Gastroenterol 2010; 45 (1): 93-99.

Received February 5, 2017. Accepted March 11, 2017. 